\title{
The Emergence of ADHD Comorbidity in School- Aged Children with ASD Impacts Resting-State Brain Networks Dynamics
}

\author{
Aurélie Bochet ( $\square$ aurelie.bochet@unige.ch ) \\ University of Geneva Faculty of Medicine: Universite de Geneve Faculte de Medecine \\ https://orcid.org/0000-0002-5476-6170 \\ Fiona Journal \\ University of Geneva Faculty of Medicine: Universite de Geneve Faculte de Medecine \\ Holger Franz Sperdin \\ University of Geneva Faculty of Medicine: Universite de Geneve Faculte de Medecine \\ Marie Schaer \\ University of Geneva Faculty of Medicine: Universite de Geneve Faculte de Medecine
}

\section{Research}

Keywords: EEG, microstates, children, ASD, ADHD, comorbidity

Posted Date: December 20th, 2021

DOI: https://doi.org/10.21203/rs.3.rs-1156053/v1

License: (c) (i) This work is licensed under a Creative Commons Attribution 4.0 International License. Read Full License 


\section{Abstract}

Background: EEG microstates are defined in the literature as quasi-stable topographies of the electric fields in the ongoing EEG, lasting approximately 100 milliseconds and representing the sub-second coherent activation within global functional brain networks. Recently, we found early alterations in the spatio-temporal dynamics and syntax of brain states in toddlers and preschoolers with autism spectrum disorders (ASD) compared to their typically developing (TD) peers.

Method: Here, we investigated how these alterations evolve over time and how they are impacted by the emergence of comorbid attention deficit/hyperactivity disorder (ADHD) in ASD among 49 school-aged children (19 children with ASD-only, 15 children with ASD+ADHD, and 15 TD children).

Results: We found a decreased prevalence of all temporal parameters of microstate map B in schoolaged children with ASD compared to TD peers. Children with ASD+ADHD had a significantly lower prevalence of map B compared to children with ASD-only. Moreover, children with ASD+ADHD had a higher prevalence in time coverage and occurrence for microstate map A than children with ASD-only and TD children. Map E of the ASD+ADHD group has a weaker spatial correlation with map E of ASD-only and TD groups. Moreover, the GEV of map E was significantly decreased for the ASD+ADHD group compared to the ASD-only group. We found that the transition probabilities between maps of the ASD+ADHD group, particularly for map B, differed from ASD-only and TD groups. Exploratory longitudinal analysis showed an increase in all temporal parameters of map B in TD children but a decrease in children with ASD, regardless of the ADHD comorbidity.

Limitations: The present study has a small sample size and does not include a group of children with ADHD-only.

Conclusion: Our results show that ADHD comorbidity in school-aged children with ASD impacts the spatial organization and the temporal dynamics of whole-brain networks. Further longitudinal studies are needed to understand better the impact of the emergence of ADHD comorbidity in ASD across ages.

\section{Background}

According to DSM-5, major diagnostic criteria for autism spectrum disorders (ASD) are impairments in communication, social interactions, and repetitive behaviors and restricted interests (1), while attention deficit/hyperactivity disorder (ADHD) is defined by symptoms of inattention and/or hyperactivity/impulsivity (1). ASD and ADHD are two of the most frequent neurodevelopmental disorders of child psychiatry, with a prevalence among children of $1.85 \%$ for ASD (2) and up to $11 \%$ for ADHD (3). Since the release of DSM-5 in 2013, the co-diagnosis of both disorders is now possible (1). This update reflects the frequent clinical co-occurrence between both disorders. Indeed, ADHD is one of the most cooccurring psychiatric disorders in ASD (4). According to the literature, up to $85 \%$ of children with ASD have ADHD symptoms $(4,5)$. Conversely, up to two-thirds of children with ADHD have ASD symptoms $(5$, 6). Several studies showed an impact of comorbid ADHD symptoms amongst children with ASD, with 
lower adaptative functioning, quality of life, and social skills (5, 7-9). Shared genetic factors, with a cross-risk between both disorders (10-12), and environmental risk factors, such as low birth weight (13) between both disorders suggest some common etiologies. Moreover, observing clinical and neuroimaging similarities between ASD and ADHD (5), recent hypotheses have emerged concerning potential shared developmental pathways and mechanisms leading to both disorders (14-17). Among imaging studies, a common feature of ASD and ADHD is whole-brain functional dysconnectivity $(18,19)$. Indeed, several studies have found connectivity particularities in resting-state brain networks (RSNs), such as the Default Mode Network (DMN) for both disorders separately (for a review, see (20)) and when they co-occur together (21). However, underlying mechanisms leading to ASD-ADHD comorbidity are not entirely understood.

Although ASD are now reliably diagnosed around 2 years old (22), the diagnosis of ADHD is usually confirmed through clinical observations of symptoms after 6 years of age (23). The potential for shared overlapping causal mechanisms between ASD and ADHD suggests the possibility for early neurobiological markers of their comorbidity. This would help early and accurate diagnosis as neurobiological markers may be more easily and precisely measured than clinical phenotypes and present before clinical symptoms emerge. Electroencephalography (EEG) is a valuable tool to understand particularities of brain function in neurodevelopmental disorders because of its higher relative tolerance for movement, higher temporal resolution, and ease of use in daily practice with children with ASD compared to magnetic resonance imaging (MRI).

During the last two decades, a growing body of work has focused on high-density EEG microstate analysis (24). Microstate analysis is a broad-band multichannel EEG approach to examine differences in whole-brain neuronal networks' spatial organization and temporal dynamics. Microstates are quasi-stable topographies of the brain electric field at the scalp with short-lasting periods (around 100 milliseconds), representing the subsecond coherent activation within global functional brain networks (24). This EEG method allows investigating the dynamic fluctuation of RSNs at a large-scale level in the pediatric population with a much higher temporal resolution than MRI (25). Initially, Koenig and colleagues (26) described four prototypical topographies labeled microstate maps $A, B, C$, and $D$ that have been retained through the literature (24).

Resting-state microstate analysis has been well explored in healthy controls and several psychiatric pathologies, particularly schizophrenia. Indeed, the deviant dynamic of EEG microstate maps $C$ and D are suggested to represent a promising endophenotype candidate for schizophrenia (27). Recently, we investigated spatio-temporal dynamics of whole-brain neuronal networks in 113 toddlers and preschoolers under 6 years old (66 with ASD) using an EEG microstate approach (28). We found differences in the spatio-temporal dynamics and syntax of brain states in toddlers and preschoolers with ASD compared to TD peers.

Here we used an EEG microstate approach to study how these alterations evolve over time and how they are impacted by the emergence of comorbid ADHD in ASD among 49 school-aged children (19 children 
with ASD-only, 15 children with ASD+ADHD, and 15 TD children). Here, we used EEG microstate approach to examine differences in the spatial organization and temporal dynamic of resting-state brain networks in a sample of school-aged children with ASD with or without ADHD and TD peers ( $N=49,34$ children with ASD, 8 years of age on average). We hypothesized that children with ASD+ADHD would show alterations in the temporal parameters and syntax of microstate maps compared to ASD-only and TD groups. To investigate the impact of the emergence of ADHD in children with ASD better, we performed longitudinal analysis using previous recordings of 21 participants with ASD, and 14 TD acquired when they were younger (4 years of age on average).

\section{Method}

\section{Participants}

Since we had more boys than girls in the ASD group, and to avoid a gender bias in this study, we decided to focus on males only. In total, 53 participants were recruited for the experiment. We managed to put the cap on 50 participants (34 ASD and 23 TD) as 3 participants with ASD refused it. We excluded one further participant after checking the data quality before starting analysis because of too many movement-related artifacts and insufficient amounts of epochs available for subsequent analysis (see below for detailed EEG preprocessing procedure). As a result, 49 boys were included in the final sample: 19 children with ASD-only (mean age 7.59 years old \pm 1.16 , range 6.25-10.28), 15 children with ASD+ADHD (mean age 8.45 years old \pm 1.32 , range 6.44-10.43) and 15 TD peers (mean age 8.73 years old \pm 1.51 , range $6.24-11.30$ ). Groups differ by age ( $p$-value $=0.040$ ). This difference is driven mainly by a tendency for the ASD-only group to be younger than the TD group but without a significant difference regarding post hoc pairwise comparisons adjusted with Bonferroni correction ( $p$-value $=0.050$ ). To minimize potential age bias, age was considered as a covariate in further analyses.

All participants were recruited as a part of the longitudinal Autism Brain and Behavior (ABB) lab's cohort (28-31). The cohort includes children from 3 months old to 12 years old with ASD and TD peers. Children with ASD were recruited in the cohort through local ASD-specialized therapeutic centers and parent associations. Children were included in the ASD group if the clinical diagnosis of ASD was confirmed by exceeding the threshold limit on Autism Diagnostic Observation Schedule, second version (ADOS-2)(32). Experienced medical doctors or psychologists working in the research team and specialized in ASD identification performed and scored the ADOS-2. To ensure comparability between children's evaluations, according to age and language level, we used the ADOS-2 calibrated severity scores (total, social affect and restricted and repetitive behaviors severity scores)(33). The mean of total severity score at ADOS-2 was $7.11 \pm 2.08$ for children with ASD-only and $8.13 \pm 1.19$ for children with ASD+ADHD. Both groups did not differ in severity of ASD symptoms ( $p$-value $=0.098$ ).

For the TD group, children were recruited through announcements in the Geneva community. They were also assessed with ADOS-2 to ensure the absence of ASD symptoms prior their inclusion in the TD group of the ABB lab's cohort. All TD participants had a minimal ADOS-2 severity score of 1 prior their inclusion. 
Children were not included in the TD group if they presented any neurological or developmental conditions and learning disabilities according to parents' interview and questionnaire, or if they had a sibling or first-degree parent diagnosed with ASD.

To assess potential ADHD comorbidity in children with ASD, we used the ADHD Child Evaluation (ACE) (34), a diagnostic interview of ADHD with parents for children between 5 and 18 years old. The ACE explores nine symptoms of attention deficit and nine symptoms of hyperactivity/impulsivity. Experienced medical doctors or psychologists working in the research team confirmed the ADHD comorbidity if children met the DSM-5 diagnostic criteria based on the ACE and/or clinical observations. Of the 15 participants with comorbid ADHD, 9 participants met the diagnostic criteria for predominantly inattentive presentation ( $\geq 6$ criteria of attention deficit), 3 participants for predominantly hyperactive/impulsive presentation ( $\geq 6$ criteria of hyperactivity/impulsivity), and 3 participants for combined presentation ( $\geq 6$ criteria of attention deficit and $\geq 6$ criteria of hyperactivity/impulsivity).

Only one participant with ASD+ADHD was taking medication (Lysdexamfetamine) at the time of the study. To avoid bias, this child refrained from taking his medication for 24 hours before assessments. Two more boys with ASD+ADHD had previously received stimulants (Methylphenidate) for a short period of time but stopped it several months before the study. Therefore, no participant was taking any medication during assessments.

To investigate other comorbid symptoms that could be present in children with ASD, we used the KiddieSchedule for Affective Disorders and Schizophrenia (K-SADS), present version (35). The K-SADS is a semi-structured parents' interview facilitating the diagnosis of past and current episodes of psychopathology in children and adolescents between 6 and 18 years old according to DSM- 5 criteria. Regarding K-SADS interview, 1 participant with ASD met the diagnostic criteria for a comorbid diagnosis of specific phobia and 1 participant with ASD+ADHD met the diagnostic criteria for a generalized anxiety disorder.

Considering the age of participants, for all children finally included in the study, cognitive functioning was assessed using the Wechsler intelligence scale for school-aged children, fifth version (WISC-V)(36). The WISC-V is a standardized assessment used to measure intellectual quotient for children from 6 to 16 years old. Information about intellectual functioning includes five verbal and non-verbal indexes: visual comprehension index, visual spatial index, fluid reasoning index, working memory index, processing speed index. The full-scale intelligence quotient (FSIQ) is referred to as an estimate of overall intelligence. FSIQ was invalid for two participants because of behavioral problems and receptive language difficulties during the assessments. The mean of FSIQ was $99.8 \pm 14.7$ for children with ASD-only and $90.5 \pm 10.4$ for children with ASD+ADHD. The ASD+ADHD group tended to have a lower cognitive level than the ASDonly group $(\mathrm{p}$-value $=0.053)$.

All participants' characteristics are summarized in Table 1. 
Table 1

Participants' characteristics for ASD-only, ASD+ADHD and TD groups.

\begin{tabular}{|c|c|c|c|c|}
\hline & ASD-only & ASD+ADHD & TD & P-values \\
\hline \multicolumn{5}{|l|}{$N$} \\
\hline Participants & 19 Males & 15 Males & 15 Males & \\
\hline \multicolumn{5}{|c|}{ Mean, SD (range) } \\
\hline \multirow[t]{2}{*}{ Age } & $7.59 \pm 1.16$ & $8.45 \pm 1.32$ & $8.73 \pm 1.51$ & $0.040 *$ \\
\hline & $(6.25-10.28)$ & $(6.44-10.43)$ & $(6.24-11.30)$ & \\
\hline \multirow[t]{2}{*}{ ADOS-2 - Total } & $7.11 \pm 2.08$ & $8.13 \pm 1.19$ & - & 0.098 \\
\hline & $(4-10)$ & $(6-10)$ & & \\
\hline \multirow[t]{2}{*}{ WISC-V - FSIQ } & $99.8 \pm 14.7$ & $90.5 \pm 10.4$ & -- & 0.053 \\
\hline & $(71-118)$ & $(71-105)$ & & \\
\hline
\end{tabular}

$A S D=$ Autism Spectrum Disorders; $A D H D=$ Attention Deficit/Hyperactivity Disorder; $T D=$ Typically Developing; ADOS-2 = Autism Diagnostic Observation Schedule, second version; WISC-V = Wechsler Intelligence Scale for school-aged Children, fifth version; FSIQ = Full Scale Intelligence Quotient; *significant $p$-values $<0.05$.

\section{Acquisitions and preprocessing of EEG data}

We conducted the experiment in a quiet room and children were seated on a comfortable chair. The experimenter first measured the circumference of the head and then the cap of the corresponding size was prepared (left for 5 minutes in salted water) and placed on the child's head. During all experiment, we showed to children an age-appropriate animated cartoon of their choice to best capture their attention. The EEG expert visually ensured that the cap was accurately placed. Impedances were measured and electrodes were adjusted to keep the lowest possible values (below $50 \mathrm{kOhm}$ ) before starting the recording. We used the Hydrocel Geodesic Sensor Net (HCGSN, Electrical Geodesics, USA) with 129 scalp electrodes at a sampling frequency of $1000 \mathrm{~Hz}$ and with onscreen filters set at $0.1-100 \mathrm{~Hz}$ during online recording. Around seven minutes of spontaneous EEG recordings were acquired for all the participants included in the study.

Data pre-processing and microstate analysis were done using Cartool (http://sites.google.com/site/cartoolcommunity/) and Matlab (Natick, MA). First, we down-sampled to 110 electrodes montage to exclude electrodes on the cheek and the neck since those are often contaminated with muscular-related artifacts. Data were filtered between 1 and $40 \mathrm{~Hz}$ using Butterworth filters and a $50 \mathrm{~Hz}$ notch filter was applied. Each file was visually inspected to detect periods of movement-related artifacts and then these periods were excluded. We then performed Independent 
Component Analysis (ICA) on all the data to identify and remove the components related to eye movement artifacts (eye blinks, saccades) $(37,38)$. Electrodes with substantial noise were interpolated using 3D spline interpolation for each recording. For each recording, less than $15 \%$ of the total electrodes of the recording were interpolated. A spatial filter was applied, the cleaned data were down sampled to $125 \mathrm{~Hz}$ and were then re-referenced to the average reference. Finally, all data were reinspected one last time by an EEG expert (AB) to ensure that no artifacts had been missed.

\section{Cross-sectional microstate analysis}

After preprocessing, we individually applied a k-means cluster analysis to the EEG recording of each subject to estimate the optimal topographies explaining the data. The k-means cluster analysis applied a meta-criterion that includes seven independent criteria. See (39) and (40) for a detailed description of these criteria. The clustering was applied only at local maxima of the Global Field Power (GFP), calculated as the standard deviation of all electrodes at a given time point, in order to include only time points of highest signal-to-noise ratio (see (41) for formulas). During the procedure, the polarity of the maps was ignored.

Then, we applied a second k-means cluster analysis at the group level, across all participants with ASDonly, participants with ASD+ADHD and TD participants separately, to obtain the group cluster maps. We then identified and extracted the five prototypical maps that best described the dataset of the three groups separately. We chose a priori to use five microstate maps for analyses in order to allow comparison with our previous study among toddlers and preschoolers with ASD and their TD peers (28).

We computed Pearson's spatial correlation for each of the five microstate maps to ensure that those five prototypical microstate maps were highly similar across the three groups. High spatial correlation coefficients indicated that the microstate classes were indeed similar amongst the three groups, excepted for map E. We therefore used those three templates of five microstate maps extracted from the three groups separately for further analysis. The three templates were fitted back to the original EEG of each subject, depending on their group. During the fitting, spatial correlation was calculated between the cluster maps and each individual data point. Then each data point was labeled with the cluster map that showed the highest correlation, ignoring polarity of the maps. Data points that did not correlate more than 50 percent with a given group cluster map were marked as unlabeled. Only labeled data points were included in the analysis as unlabeled data points and periods marked as artifacts during the preprocessing were excluded. For all participants individually, more than $50 \%$ of their EEG recording was labeled with one of the five microstate maps.

\section{Statistical analysis}

We investigated group differences between the four temporal parameters of each microstate maps using one-way ANCOVA, with age as a covariate. We then applied post hoc pairwise comparisons with Bonferroni correction. 
We analyzed the transition probabilities from each EEG microstate to any other using Markov chains. We divided the observed probabilities by the expected probabilities to account for the variability in occurrence of the maps as previously described in the literature $(40,42,43)$. The transition probabilities between microstate maps were compared between the three groups (ASD-only, ASD+ADHD, TD groups) using oneway ANOVA and post hoc pairwise comparisons adjusted with Bonferroni correction.

\section{Longitudinal analysis}

To understand the evolution of microstate maps' temporal parameters across age better, we performed exploratory longitudinal analysis using mixed model regression analysis with Matlab (Natick, MA). Among the 34 school-aged children with ASD with or without ADHD, 21 children had a previous 5-minute resting-state recording already acquired during a precedent visit in the lab. In addition, 14 children of the 15 school-aged TD children had a previous recording available (see Table 2 for preschoolers' characteristics). In total, 84 recordings were included in the longitudinal analysis.

Table 2

Preschoolers' characteristics for ASD with or without later ADHD, and TD groups.

\begin{tabular}{|llll|}
\hline & ASD & TD & P-values \\
\hline N & & & \\
\hline Participants & 21 Males & 14 Males & \\
\hline Mean, SD (range) & & & \\
\hline Age & $4.15 \pm 1.19$ & $3.77 \pm 1.28$ & 0.499 \\
& $(1.75-6.50)$ & $(1.72-5.45)$ & \\
\hline
\end{tabular}

Participants who have a previous resting-state EEG recordings acquired during a precedent visit in the longitudinal protocol. $A S D=$ Autism Spectrum Disorders; $A D H D=$ Attention Deficit/Hyperactivity Disorder; $T D=$ Typically Developing .

The preprocessing procedure and the microstate analysis were done exactly as described above for the preschoolers' recordings. The only difference is that we performed microstate analysis on two groups separately: 21 preschoolers with ASD with or without later ADHD and 14 TD peers. Indeed, we did not split the ASD group between ASD-only and ASD+ADHD children, as at that time, the diagnosis of comorbid ADHD was not confirmed. As for school-aged children, the five dominant maps template were extracted for both groups separately and were used for the back-fitting procedure to compute the four temporal parameters (GEV, mean duration, time coverage, occurrence). We then focused on microstate map $B$, as we obtained a significant group difference in our previous study among preschoolers and the present study. 
We ran the mixed model regression analysis using the longitudinal values of temporal parameters extracted from 21 preschoolers with ASD with or without later ADHD, 14 TD preschoolers, 19 school-aged children with ASD-only, 15 school-aged children with ASD+ADHD, and 15 school-aged TD children. We computed the group effect and interaction for the three groups (ASD-only, ASD+ADHD and TD). Then, to measure the impact of ADHD comorbidity in children with ASD, we ran the mixed model regression analysis only with both groups of children with ASD (ASD-only, ASD+ADHD).

\section{Results}

\section{Cross-sectional microstate analysis}

We selected the five dominant maps template extracted from the k-means cluster analysis across the three school-aged groups, which explained 81.7, 81.6 and 84.9 percent of the total variance for the ASDonly group, the ASD+ADHD group and the TD group respectively (Figure 1). The templates of five dominant maps extracted from the three groups separately were highly similar (see Figure 2 for Pearson's spatial correlations) and were retained for further analysis.

The three templates of five dominant maps were visually highly similar to those we found in our previous study among 113 preschoolers with ASD and their TD peers (28) and to those previously described in the literature, and were therefore labeled maps A, B, C, D, and E accordingly.

When looking for differences between the three groups in temporal parameters for the five maps (see Figure 3), covarying for age participants, we found differences in all temporal parameters (GEV, mean duration, time coverage, occurrence) of map B (all $p<0.001$ ). Moreover, we found significant differences for time coverage ( $p$-value $=0.006)$ and occurrence $(p$-value $=0.004)$ of map A between the three groups. Regarding post hoc pairwise comparisons, adjusted with Bonferroni correction, we found a decreased prevalence of map $B$ in children with ASD (ASD only and ASD+ADHD) compared to their TD peers ( $p$ values ranging from $<0.001$ to 0.007 ). Moreover, the $A S D+A D H D$ group had decreased time coverage ( $p$ value $=0.002)$ and occurrence $(p$-value $=0.001)$ for map $B$ in comparison to the ASD-only group. For map $A$, the ASD+ADHD group had increased time coverage and occurrence compared to the ASD-only group ( $p$-values $=0.042$ and 0.048 respectively) and TD group ( $p$-values $=0.008$ and 0.005 respectively). There was no significant difference for time coverage and occurrence of map A between the ASD-only group and the TD group. Finally, the GEV of map E was significantly decreased for ASD+ADHD group compared to ASD-only group ( $p$-value $=0.015)$.

There was no significant difference in temporal parameters for map $C$ and $D$ between the three groups ( $p$ values ranging from $p$-value $=0.101$ to $p$-value $=0.819$ ) .

See Table 3 for temporal parameters values for both groups. 
Table 3

Temporal parameters for the five maps of ASD-only, ASD+ADHD and TD groups.

\begin{tabular}{|c|c|c|c|c|c|c|}
\hline & & ASD-only & ASD+ADHD & TD & \multicolumn{2}{|c|}{ One-way ANCOVA } \\
\hline \multicolumn{2}{|c|}{ Temporal parameters } & Mean $\pm S D$ & & & $F$ & $p$-values \\
\hline \multirow[t]{4}{*}{$\begin{array}{l}\text { Map } \\
\text { A }\end{array}$} & $\begin{array}{l}\text { Global Explained } \\
\text { Variance }\end{array}$ & $\begin{array}{l}0.07021 \pm \\
0.01727\end{array}$ & $\begin{array}{l}0.07921 \pm \\
0.01687\end{array}$ & $\begin{array}{l}0.06943 \pm \\
0.01557\end{array}$ & 1.609 & 0.211 \\
\hline & $\begin{array}{l}\text { Mean Duration } \\
(\mathrm{ms})\end{array}$ & $68.81 \pm 2.103$ & $70.60 \pm 3.336$ & $69.24 \pm 3.144$ & 1.370 & 0.264 \\
\hline & Time Coverage (\%) & $15.36 \pm 2.343$ & $18.01 \pm 2.797$ & $15.28 \pm 2.394$ & 5.766 & $0.006^{\star \star}$ \\
\hline & Occurrence $\left(\mathrm{s}^{-1}\right)$ & $\begin{array}{l}1.982 \pm \\
0.2550\end{array}$ & $\begin{array}{l}2.238 \pm \\
0.2604\end{array}$ & $\begin{array}{l}1.958 \pm \\
0.2135\end{array}$ & 6.147 & $0.004^{\star \star}$ \\
\hline \multirow[t]{4}{*}{$\begin{array}{l}\text { Map } \\
\text { B }\end{array}$} & $\begin{array}{l}\text { Global Explained } \\
\text { Variance }\end{array}$ & $\begin{array}{l}0.07808 \pm \\
0.01676\end{array}$ & $\begin{array}{l}0.06366 \pm \\
0.01691\end{array}$ & $\begin{array}{l}0.1042 \pm \\
0.01914\end{array}$ & 20.190 & $<_{0.001 * \star \star}$ \\
\hline & $\begin{array}{l}\text { Mean Duration } \\
(\mathrm{ms})\end{array}$ & $70.41 \pm 1.898$ & $68.08 \pm 2.900$ & $73.70 \pm 3.008$ & 17.426 & $<.001^{\star \star * *}$ \\
\hline & Time Coverage (\%) & $17.69 \pm 2.342$ & $14.15 \pm 2.407$ & $21.08 \pm 3.045$ & 26.716 & $<.001^{\star * *}$ \\
\hline & Occurrence $\left(\mathrm{s}^{-1}\right)$ & $\begin{array}{l}2.216 \pm \\
0.2396\end{array}$ & $\begin{array}{l}1.848 \pm \\
0.2518\end{array}$ & $\begin{array}{l}2.494 \pm \\
0.2741\end{array}$ & 24.468 & 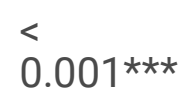 \\
\hline \multirow[t]{4}{*}{$\begin{array}{l}\text { Map } \\
\text { C }\end{array}$} & $\begin{array}{l}\text { Global Explained } \\
\text { Variance }\end{array}$ & $\begin{array}{l}0.2167 \pm \\
0.06129\end{array}$ & $\begin{array}{l}0.2322 \pm \\
0.06688\end{array}$ & $\begin{array}{l}0.2350 \pm \\
0.05848\end{array}$ & 0.328 & 0.722 \\
\hline & $\begin{array}{l}\text { Mean Duration } \\
\text { (ms) }\end{array}$ & $83.08 \pm 6.829$ & $87.24 \pm 11.19$ & $84.51 \pm 6.677$ & 1.022 & 0.368 \\
\hline & Time Coverage (\%) & $29.42 \pm 6.098$ & $33.68 \pm 7.838$ & $30.18 \pm 5.421$ & 1.832 & 0.172 \\
\hline & Occurrence $\left(\mathrm{s}^{-1}\right)$ & $\begin{array}{l}2.993 \pm \\
0.3335\end{array}$ & $\begin{array}{l}3.213 \pm \\
0.2504\end{array}$ & $\begin{array}{l}3.019 \pm \\
0.2788\end{array}$ & 2.41 & 0.101 \\
\hline \multirow[t]{4}{*}{$\begin{array}{l}\text { Map } \\
\text { D }\end{array}$} & $\begin{array}{l}\text { Global Explained } \\
\text { Variance }\end{array}$ & $\begin{array}{l}0.08541 \pm \\
0.03277\end{array}$ & $\begin{array}{l}0.09431 \pm \\
0.03630\end{array}$ & $\begin{array}{l}0.08001 \pm \\
0.03456\end{array}$ & 0.649 & 0.527 \\
\hline & $\begin{array}{l}\text { Mean Duration } \\
(\mathrm{ms})\end{array}$ & $71.87 \pm 5.241$ & $72.41 \pm 4.193$ & $71.28 \pm 4.708$ & 0.201 & 0.819 \\
\hline & Time Coverage (\%) & $18.97 \pm 4.771$ & $18.00 \pm 4.545$ & $17.02 \pm 5.348$ & 0.515 & 0.601 \\
\hline & Occurrence $\left(\mathrm{s}^{-1}\right)$ & $\begin{array}{l}2.295 \pm \\
0.3655\end{array}$ & $\begin{array}{l}2.173 \pm \\
0.4247\end{array}$ & $\begin{array}{l}2.083 \pm \\
0.5208\end{array}$ & 0.755 & 0.476 \\
\hline \multirow[t]{2}{*}{$\begin{array}{l}\text { Map } \\
E\end{array}$} & $\begin{array}{l}\text { Global Explained } \\
\text { Variance }\end{array}$ & $\begin{array}{l}0.09501 \pm \\
0.02094\end{array}$ & $\begin{array}{l}0.06608 \pm \\
0.02564\end{array}$ & $\begin{array}{l}0.07931 \pm \\
0.03049\end{array}$ & 4.344 & $0.019 *$ \\
\hline & $\begin{array}{l}\text { Mean Duration } \\
(\mathrm{ms})\end{array}$ & $72.00 \pm 2.832$ & $70.15 \pm 4.259$ & $70.47 \pm 4.048$ & 0.998 & 0.377 \\
\hline
\end{tabular}




\begin{tabular}{|llllll|}
\hline & ASD-only & ASD+ADHD & \multicolumn{2}{l|}{ TD } & \multicolumn{2}{c|}{ Oneway ANCOVA } \\
\hline Time Coverage (\%) & $18.56 \pm 3.018$ & $16.16 \pm 4.940$ & $16.44 \pm 5.172$ & 1.097 & 0.343 \\
\hline Occurrence $\left(\mathbf{s}^{-1}\right)$ & $2.267 \pm$ & $2.014 \pm$ & $2.043 \pm$ & 1.153 & 0.325 \\
& 0.2710 & 0.5330 & 0.5361 & & \\
\hline
\end{tabular}

$A S D=$ Autism Spectrum Disorders; $A D H D=$ Attention Deficit/Hyperactivity Disorder; $T D=$ Typically Developing; *significant $p$-values $<0.05$; **significant $p$-values $<0.01$; ***significant $p$-values $<0.001$.

\section{Transitions probabilities}

Using a one-way ANOVA, we found different transitions probabilities between the three groups regarding transitions from map A to map $B(p$-value $=0.002), C(p$-value $=0.002)$, and $D(p$-value $=0.004)$, from map $C$ to map $A$ ( $p$-value $<0.001)$, and $B(p$-value $=0.002)$, and from map $D$ to $B(p$-value $=0.003)$. Regarding the pairwise comparisons, corrected with Bonferroni for multiple comparisons, there were no significant difference in transitions probabilities between ASD-only and TD groups. However, we found decreased transitions from maps $A, C$ and $D$ to map $B$ for ASD+ADHD group compared to both ASD-only and TD groups. Moreover, we found increased transitions from map $C$ to $A$, for ASD+ADHD group compared to both ASD-only and TD groups, and increased transitions from map A to $C$ for ASD+ADHD group compared to ASD-only group.

See Figure 4 for transitions probabilities statistics values.

\section{Longitudinal analysis}

When looking for map B (Figure 5) temporal parameters evolution across age using mixed model analysis, we found significant group effect and significant interaction between the three groups for all parameters (all p-values group effect $<0.001$ and all $p$-values interaction $<0.001$ ). Indeed, we measured a decrease in all parameters for children with ASD (ASD-only and ASD+ADHD groups) but an increase in all parameters for TD children (Figure 6). The difference between group was driven mainly by the difference between TD and ASD groups regardless of ADHD comorbidity. Indeed, regarding pairwise comparisons between ASD-only and ASD+ADHD group, there was only a tendency for group effect for occurrence of map B ( $p$-value $=0.0503)$. However, there was no group effect for other temporal parameters ( $p$-values GEV $=0.494$, mean duration $=0.360$, time coverage $=0.106)$ nor interaction ( $p$-values GEV $=0.796$, mean duration $=0.942$, time coverage $=0.435$, occurrence $=0.2244$ ).

\section{Discussion}

The present study explored the spatial organization and temporal dynamics of resting-state brain networks and their evolution over time in a sample of school-aged children with ASD with or without ADHD and TD peers. We extracted a five maps template for each of the three groups separately. Maps 
were highly similar to those extracted from the preschoolers' sample in our previous study and were therefore labeled accordingly maps A, B, C, D, and E (28). When comparing temporal parameters between the three groups with cross-sectional analysis, we found a decreased prevalence of all temporal parameters of map B in school-aged children with ASD (ASD-only and ASD+ADHD groups) compared to TD peers. Children with ASD+ADHD had a significantly lower prevalence of map $B$ compared to children with ASD-only. Moreover, children with ASD+ADHD had a higher prevalence in time coverage and occurrence for microstate map A than children with ASD-only and TD children. In addition to a weaker spatial correlation for map E of ASD+ADHD group, the GEV of map E was significantly decreased for ASD+ADHD group compared to ASD-only group. Moreover, we found a specific dynamic pattern for children with ASD+ADHD regarding the transition probabilities between each map. Indeed, we found no significant difference between ASD-only and TD groups. However, we found decreased transitions probabilities from maps $A, C$, and $D$ to map $B$ for ASD+ADHD group compared to both ASD-only and TD groups. We also found increased transitions from map $C$ to $A$ for ASD+ADHD group compared to ASDonly and TD groups, and increased transitions from map $A$ to $C$ for ASD+ADHD group compared to ASDonly group.

Altogether, those results showed that school-aged children with ASD and comorbid ADHD showed a specific pattern of microstate maps topography, temporality, and dynamic compared to children with ASD-only and TD peers. These specificities may represent the alterations in RSNs connectivity leading to ADHD-ASD comorbidity reported in the MRI literature $(21,44)$. To understand the functional significance of microstates, the seminal EEG-fMRI study of Britz and colleagues associated each microstate with a specific RSN (45).In their study, map 1, corresponding to our map A, was related to negative activation in bilateral superior and middle temporal gyri (45). The authors linked those areas with phonological processing. In parallel, map 2, corresponding to our map B, was related to activation in bilateral extrastriate visual areas associated with the visual network. Britz and colleagues initially linked microstate map $\mathrm{C}$ with activation in the anterior cingulate cortex, bilateral inferior frontal gyri, and the insula (45), referred to the salience network (46). More recently, microstate map $C$ has been related to the Default Mode Network $(\mathrm{DMN})(40,47)$, with bilateral activity in the lateral part of the parietal lobe and middle temporal gyrus found in EEG-fMRI study of Bréchet and colleagues (40). Map E from our dataset visually corresponds to microstate map 4 described in Britz and colleagues' study, with a fronto-central maximum (45). The authors found a correlation between map 4 and activation in the right-lateralized dorsal and ventral areas of the frontal and parietal cortex (45). They linked those ventral and dorsal fronto-parietal areas to attention processes such as detection of stimuli and orientation of attention, respectively $(45,48)$. Using functional MRI, Yerys and colleagues highlighted a correlation between decreased connectivity within salience/ventral attention sub-network and between frontoparietal subnetworks with ADHD symptoms in children with ASD (44). Moreover, Wang and colleagues showed an impact of the comorbidity between ASD and ADHD compared to ASD alone on DMN connectivity (21). They found an increased intra-connectivity of the bilateral posterior cingulate cortex of the DMN and increased interconnectivity between the DMN and somatomotor networks. 
To the best of our knowledge, only a few other studies using a microstate approach in individuals with ASD have been published (49-55). Across resting-state studies, D'Croz-Baron and colleagues found six microstate maps that best described their dataset during an eyes-closed condition (49). They found an increased occurrence for microstate maps $B$ and $E$ in young adults with ASD compared to their TD peers. There was a trend for map $C$ being more present in their control group. Jia and $\mathrm{Yu}$ also highlighted a microstate map B more prevalent in occurrence and time coverage, a decreased map A regarding the mean duration, and a decreased map $C$ regarding the mean duration and time coverage when comparing 5- to 18-year-old children with ASD to their TD peers (52). Finally, Nagabhushan Kalburgi and colleagues found an increased mean duration but a decreased occurrence in microstate map $C$ in children (8-14 years old) with ASD compared to TD in an eyes-closed condition (54). In the study of Jia and Yu, none of the ASD children had ever received a diagnosis of comorbid ADHD, but some participants were taking stimulants medication (52). The medication was interrupted for the study. In Nagabhushan and colleagues' study, 6 children with ASD were taking various ADHD medications and took their usual dose when assessed for the study (54).

Our previous study among toddlers and preschoolers with ASD and TD peers found opposite results regarding microstate map $B(28)$. We found an increased prevalence for all temporal parameters in toddlers and preschoolers with ASD compared to their TD peers (28). Our exploratory longitudinal analysis indicated a decrease in all parameters for children with ASD (ASD-only and ASD+ADHD groups) but an increase in all parameters for TD children. The difference between groups was mostly driven by the difference between TD and ASD groups regardless of ADHD comorbidity except for the occurrence, which showed a tendency for a group effect between ASD-only and ASD+ADHD. The reversal in the prevalence of map B in ASD may represent the developmental shift in functional connectivity suggested by Uddin and colleagues, 2013 (56). The authors highlighted in the literature that studies exploring resting-state fMRI of young children with ASD mostly found brain hyperconnectivity while hypoconnectivity was prevalent in an older population with ASD (adolescents, adults). The shift may be accentuated by the emergence of comorbidity such as ADHD.

Few studies have investigated the evolution of microstate maps parameters across age among the general population, with divergent findings $(43,57,58)$. On the one hand, Tomescu and colleagues explored the evolution of mean duration and occurrence of four microstate maps (A to $D$ ) in eyes-closed resting-state condition, starting from 6 years old (43). They found a general tendency for an increased microstates mean duration with age except for microstate map C. Indeed, in TD boys, microstate map C showed a nonlinear evolution with an increase in mean duration from childhood to adolescence and later on from 20 to 60 years but showed a tendency of a decreased mean duration from adolescence to young adulthood. In addition, the authors highlighted a trend for decreased microstates occurrence across ages, driven mainly by a decrease in map $D$ in male children and adolescents. On the other hand, Koenig and colleagues found a general decrease in microstates mean duration across age in the TD population, except for microstate map $\mathrm{C}$, which also presented a nonlinear evolution with a maximal mean duration around late adolescence (57). Koenig and colleagues concluded that children between 6 and 12 years old showed relatively stable microstates temporal parameters. However, we are the first study exploring the 
effect of age on microstate maps parameters before 6 years old. We focused on microstate map B but considering the critical developmental period for brain changes during the first years of life, we assume spatio-temporal dynamics variations in parameters of other microstate maps during this period. Moreover, we are the first study investigating the evolution of microstate maps parameters across age using longitudinal data.

\section{Limitations}

The major limitation of this study is the small sample size. Potential co-occurring conditions play a role in the heterogeneity in ASD, and poor recognition of co-morbidities probably contributes to the divergence in the literature on ASD. Therefore, we decided to split the ASD group into two subgroups (ASD-only, ASD+ADHD) to account for their frequent clinical co-occurrence. The small sample size is also a limitation to longitudinal analyses, which are therefore only exploratory. Indeed, we used two large age windows (preschoolers versus school-aged children), which do not allow us to have a clear picture of the microstate map B evolution across age. A better way to explore longitudinal data might be by doing a sliding window analysis of an age interval of one year. This would allow following more precisely the evolution of microstates maps even if the evolution is not linear. The second major limitation of the present study is that we don't have an ADHD-only group. Moreover, no study has yet explored microstate analysis among ADHD individuals to the best of our knowledge. Therefore, we cannot conclude that the alterations found in the ASD+ADHD group are the addition of the modifications in ASD and ADHD separately or if there is a synergic effect. Finally, there is a difference between the three age groups, driven by a tendency for the ASD-only group to be younger than the TD group. We covaried with age to limit the impact of age on group comparisons. Still, results should be taken with caution. Further longitudinal analyses with more significant sample are needed to understand better the evolution of microstate maps across age in children with ASD considering the emergence of potential comorbidities in children with ASD.

\section{Conclusions}

Only a few studies have been published using resting-state microstates analysis among children with ASD. Moreover, no study has considered the frequent clinical comorbidity between ASD and ADHD. The present study is the first to investigate the evolution of alterations in resting-state networks dynamics among children with ASD, specifically looking at the emergence of ADHD. Our results show that ADHD comorbidity in school-aged children with ASD impacts the spatio-temporal dynamics and syntax of whole-brain resting-state networks. Moreover, exploratory longitudinal analysis showed an increase in all temporal parameters of map $B$ in TD children but a decrease in children with ASD, regardless of the ADHD comorbidity across ages. Further longitudinal studies with a bigger sample size are needed to fully understand the evolution of microstates considering the impact of potential comorbidities in ASD.

\section{Abbreviations}


ACE

ADHD Child Evaluation

ADHD

attention deficit/hyperactivity disorder

ADOS-2

autism diagnostic observation schedule, second version

ASD

autism spectrum disorders

DMN

default mode network

EEG

electroencephalogram

FSIQ

Full Scale Intelligence Quotient

GEV

global explained variance

GFP

global field power

K-SADS

Kiddie-Schedule for Affective Disorders and Schizophrenia

RSNs

resting-state brain networks

TD

typically developing

VIS

visual network. WISC-V=Wechsler Intelligence Scale for school-aged Children, fifth version.

\section{Declarations}

\section{Ethics approval and consent to participate}

The Local Research Committee, the Commission Centrale d'Ethique de Recherche (CCER) in Geneva, Switzerland, approved this study and written informed consent was obtained from all children's parents prior to inclusion in the study.

Consent for publication

Not applicable.

Availability of data and materials 
The data that support the findings of this study are available from the authors upon reasonable request, but restrictions apply to the availability of these data, which were used under license for the current study, and so are not publicly available.

\section{Competing interests}

The authors declare no competing interests.

\section{Funding}

This research was supported by the National Center for Competence in Research "Synapsy", financed by the Swiss National Science Foundation (SNF, \#51NF40-185897) and by private funding by the Fondation Pôle Autisme (http://www.pole-autisme.ch/). This work was further supported by individual SNF grants to Marie Schaer (\#163859 and \#190084) and to Aurélie Bochet (\#323530-183979).

\section{Author contributions}

Conception and design of the experiment: A.B. and M.S.; acquisition of data: A.B. and F.J.; preprocessing and analysis of data: $A B$; interpretation of results: A.B, M.S. and HFS; Drafting of the manuscript: A.B.; All authors revised the manuscript critically for important intellectual content.

Acknowledgements

The authors would like to thank all the families who took part in this research and the team of the Autism Brain and Behavior lab for the collaboration.

\section{References}

1. A.P.A. Diagnostic and Statistical Manual of Mental Disorders (DSM-5®). American Psychiatric Pub; 2013. 1679 p.

2. Maenner MJ. Prevalence of Autism Spectrum Disorder Among Children Aged 8 Years - Autism and Developmental Disabilities Monitoring Network, 11 Sites, United States, 2016. MMWR Surveill Summ [Internet]. 2020 [cited 2021 May 20];69. Available from:

https://www.cdc.gov/mmwr/volumes/69/ss/ss6904a1.htm

3. Danielson ML, Bitsko RH, Ghandour RM, Holbrook JR, Kogan MD, Blumberg SJ. Prevalence of ParentReported ADHD Diagnosis and Associated Treatment Among U.S. Children and Adolescents, 2016. J Clin Child Adolesc Psychol. 2018 Mar 4;47(2):199-212.

4. Salazar F, Baird G, Chandler S, Tseng E, O'sullivan T, Howlin P, et al. Co-occurring Psychiatric Disorders in Preschool and Elementary School-Aged Children with Autism Spectrum Disorder. J Autism Dev Disord. 2015 Aug;45(8):2283-94.

5. Leitner Y. The co-occurrence of autism and attention deficit hyperactivity disorder in children - what do we know? Front Hum Neurosci. 2014;8:268. 
6. Grzadzinski R, Dick C, Lord C, Bishop S. Parent-reported and clinician-observed autism spectrum disorder (ASD) symptoms in children with attention deficit/hyperactivity disorder (ADHD): implications for practice under DSM-5. Mol Autism. 2016;7:7.

7. Factor RS, Ryan SM, Farley JP, Ollendick TH, Scarpa A. Does the Presence of Anxiety and ADHD Symptoms Add to Social Impairment in Children with Autism Spectrum Disorder? J Autism Dev Disord. 2017 Apr 1;47(4):1122-34.

8. Rao PA, Landa RJ. Association between severity of behavioral phenotype and comorbid attention deficit hyperactivity disorder symptoms in children with autism spectrum disorders. Autism. $2014 \mathrm{Apr}$ 1;18(3):272-80.

9. Sikora DM, Vora P, Coury DL, Rosenberg D. Attention-Deficit/Hyperactivity Disorder Symptoms, Adaptive Functioning, and Quality of Life in Children With Autism Spectrum Disorder. Pediatrics. 2012 Nov 1;130(Supplement 2):S91-7.

10. Ghirardi L, Brikell I, Kuja-Halkola R, Freitag CM, Franke B, Asherson P, et al. The familial coaggregation of ASD and ADHD: a register-based cohort study. Mol Psychiatry. 2018 Feb;23(2):25762.

11. Miller M, Musser ED, Young GS, Olson B, Steiner RD, Nigg JT. Sibling Recurrence Risk and Crossaggregation of Attention-Deficit/Hyperactivity Disorder and Autism Spectrum Disorder. JAMA Pediatr. 2019 Feb 1;173(2):147-52.

12. Septier M, Peyre H, Amsellem F, Beggiato A, Maruani A, Poumeyreau M, et al. Increased risk of ADHD in families with ASD. Eur Child Adolesc Psychiatry. 2019 Feb;28(2):281-8.

13. Carlsson T, Molander F, Taylor MJ, Jonsson U, Bölte S. Early environmental risk factors for neurodevelopmental disorders - a systematic review of twin and sibling studies. Dev Psychopathol. 2020 Jul 24;1-48.

14. Johnson MH, Gliga T, Jones E, Charman T. Annual research review: Infant development, autism, and ADHD-early pathways to emerging disorders. J Child Psychol Psychiatry. 2015 Mar;56(3):228-47.

15. Miller M, Austin S, losif A-M, de la Paz L, Chuang A, Hatch B, et al. Shared and distinct developmental pathways to ASD and ADHD phenotypes among infants at familial risk. Dev Psychopathol. 2020 Oct;32(4):1323-34.

16. Sokolova E, Oerlemans AM, Rommelse NN, Groot P, Hartman CA, Glennon JC, et al. A Causal and Mediation Analysis of the Comorbidity Between Attention Deficit Hyperactivity Disorder (ADHD) and Autism Spectrum Disorder (ASD). J Autism Dev Disord. 2017 Jun;47(6):1595-604.

17. Visser JC, Rommelse NNJ, Greven CU, Buitelaar JK. Autism spectrum disorder and attentiondeficit/hyperactivity disorder in early childhood: A review of unique and shared characteristics and developmental antecedents. Neurosci Biobehav Rev. 2016 Jun 1;65(Supplement C):229-63.

18. Padmanabhan A, Lynch CJ, Schaer M, Menon V. The Default Mode Network in Autism. Biol Psychiatry Cogn Neurosci Neuroimaging. 2017 Sep;2(6):476-86.

19. Uddin LQ, Dajani DR, Voorhies W, Bednarz H, Kana RK. Progress and roadblocks in the search for brain-based biomarkers of autism and attention-deficit/hyperactivity disorder. Transl Psychiatry. 
2017 22;7(8):e1218.

20. Harikumar A, Evans DW, Dougherty CC, Carpenter KLH, Michael AM. A Review of the Default Mode Network in Autism Spectrum Disorders and Attention Deficit Hyperactivity Disorder. Brain Connect. 2021 May 1;11(4):253-63.

21. Wang K, Xu M, Ji Y, Zhang L, Du X, Li J, et al. Altered social cognition and connectivity of default mode networks in the co-occurrence of autistic spectrum disorder and attention deficit hyperactivity disorder. Aust N Z J Psychiatry. 2019 Aug;53(8):760-71.

22. Guthrie W, Swineford LB, Nottke C, Wetherby AM. Early diagnosis of autism spectrum disorder: Stability and change in clinical diagnosis and symptom presentation. J Child Psychol Psychiatry. 2013 May;54(5):582-90.

23. Halperin JM, Marks DJ. Practitioner Review: Assessment and treatment of preschool children with attention-deficit/hyperactivity disorder. J Child Psychol Psychiatry. 2019;60(9):930-43.

24. Michel CM, Koenig T. EEG microstates as a tool for studying the temporal dynamics of whole-brain neuronal networks: A review. Neurolmage. 2018 Oct 15;180(Pt B):577-93.

25. Abreu R, Simões M, Castelo-Branco M. Pushing the Limits of EEG: Estimation of Large-Scale Functional Brain Networks and Their Dynamics Validated by Simultaneous fMRI. Front Neurosci [Internet]. 2020 Apr 16 [cited 2020 Jun 3];14. Available from:

https://www.ncbi.nlm.nih.gov/pmc/articles/PMC7177188/

26. Koenig T, Lehmann D, Merlo MCG, Kochi K, Hell D, Koukkou M. A deviant EEG brain microstate in acute, neuroleptic-naive schizophrenics at rest. Eur Arch Psychiatry Clin Neurosci. 1999 Aug 1;249(4):205-11.

27. Tomescu MI, Rihs TA, Roinishvili M, Karahanoglu FI, Schneider M, Menghetti S, et al. Schizophrenia patients and 22q11.2 deletion syndrome adolescents at risk express the same deviant patterns of resting state EEG microstates: A candidate endophenotype of schizophrenia. Schizophr Res Cogn. 2015 Sep;2(3):159-65.

28. Bochet A, Sperdin HF, Rihs TA, Kojovic N, Franchini M, Jan RK, et al. Early alterations of large-scale brain networks temporal dynamics in young children with autism. Commun Biol. 2021 Aug 16;4(1):968.

29. Franchini M, Wood de Wilde H, Glaser B, Gentaz E, Eliez S, Schaer M. Brief Report: A Preference for Biological Motion Predicts a Reduction in Symptom Severity 1 Year Later in Preschoolers with Autism Spectrum Disorders. Front Psychiatry. 2016 Aug 24;7.

30. Robain F, Franchini M, Kojovic N, Wood de Wilde H, Schaer M. Predictors of Treatment Outcome in Preschoolers with Autism Spectrum Disorder: An Observational Study in the Greater Geneva Area, Switzerland. J Autism Dev Disord. 2020 Mar 12;

31. Sperdin HF, Coito A, Kojovic N, Rihs TA, Jan RK, Franchini M, et al. Early alterations of social brain networks in young children with autism. eLife. 2018 Feb 27;7:e31670.

32. Lord C, Rutter M, DiLavore PC, Risi S, Gotham K, Bishop SL. Autism Diagnostic Observation Schedule (ADOS-2) (second edition). CA: Western Psychological Services. Los Angeles; 2012. 
33. Gotham K, Pickles A, Lord C. Standardizing ADOS scores for a measure of severity in autism spectrum disorders. J Autism Dev Disord. 2009 May;39(5):693-705.

34. Young S. ADHD Child Evaluation. Psychology; 2015.

35. Kaufman J, Birmaher B, Brent D, Rao U, Flynn C, Moreci P, et al. Schedule for Affective Disorders and Schizophrenia for School-Age Children-Present and Lifetime Version (K-SADS-PL): initial reliability and validity data. J Am Acad Child Adolesc Psychiatry. 1997 Jul;36(7):980-8.

36. Wechsler D. The Wechsler intelligence scale for children (5th ed.). Bloomington, MN: Bloomington: The Psychological Corporation; 2014.

37. Jung T, Makeig S, Bell AJ. Independent component analysis of electroencephalographic data. Adv Neural Inf Process Syst. 1996;145-51.

38. Jung T-P, Makeig S, Westerfield M, Townsend J, Courchesne E, Sejnowski TJ. Removal of eye activity artifacts from visual event-related potentials in normal and clinical subjects. Clin Neurophysiol. 2000 Oct 1;111(10):1745-58.

39. Custo A, Van De Ville D, Wells WM, Tomescu MI, Brunet D, Michel CM. Electroencephalographic Resting-State Networks: Source Localization of Microstates. Brain Connect. 2017;7(10):671-82.

40. Bréchet L, Brunet D, Birot G, Gruetter R, Michel CM, Jorge J. Capturing the spatiotemporal dynamics of self-generated, task-initiated thoughts with EEG and fMRI. Neurolmage. 2019 Jul 1;194:82-92.

41. Murray MM, Brunet D, Michel CM. Topographic ERP analyses: a step-by-step tutorial review. Brain Topogr. 2008 Jun;20(4):249-64.

42. Lehmann D, Faber PL, Galderisi S, Herrmann WM, Kinoshita T, Koukkou M, et al. EEG microstate duration and syntax in acute, medication-naive, first-episode schizophrenia: a multi-center study. Psychiatry Res. 2005 Feb 28;138(2):141-56.

43. Tomescu MI, Rihs TA, Rochas V, Hardmeier M, Britz J, Allali G, et al. From swing to cane: Sex differences of EEG resting-state temporal patterns during maturation and aging. Dev Cogn Neurosci. 2018 Jun;31:58-66.

44. Yerys BE, Tunç B, Satterthwaite TD, Antezana L, Mosner MG, Bertollo JR, et al. Functional Connectivity of Frontoparietal and Salience/Ventral Attention Networks Have Independent Associations With Co-occurring Attention-Deficit/Hyperactivity Disorder Symptoms in Children With Autism. Biol Psychiatry Cogn Neurosci Neuroimaging. 2019 Apr;4(4):343-51.

45. Britz J, Van De Ville D, Michel CM. BOLD correlates of EEG topography reveal rapid resting-state network dynamics. Neurolmage. 2010 Oct 1;52(4):1162-70.

46. Seeley WW, Menon V, Schatzberg AF, Keller J, Glover GH, Kenna H, et al. Dissociable Intrinsic Connectivity Networks for Salience Processing and Executive Control. J Neurosci. 2007 Feb 28;27(9):2349-56.

47. Seitzman BA, Abell M, Bartley SC, Erickson MA, Bolbecker AR, Hetrick WP. Cognitive manipulation of brain electric microstates. Neurolmage. 2017 01;146:533-43. 
48. Corbetta M, Shulman GL. Control of goal-directed and stimulus-driven attention in the brain. Nat Rev Neurosci. 2002 Mar;3(3):201-15.

49. D'Croz-Baron DF, Baker M, Michel CM, Karp T. EEG Microstates Analysis in Young Adults With Autism Spectrum Disorder During Resting-State. Front Hum Neurosci. 2019;13:173.

50. Gui A, Bussu G, Tye C, Elsabbagh M, Pasco G, Charman T, et al. Attentive brain states in infants with and without later autism. Transl Psychiatry. 2021 Mar 30;11(1):196.

51. Jan RK, Rihs TA, Kojovic N, Sperdin HF, Franchini M, Custo A, et al. Neural Processing of Dynamic Animated Social Interactions in Young Children With Autism Spectrum Disorder: A High-Density Electroencephalography Study. Front Psychiatry. 2019;10:582.

52. Jia H, Yu D. Aberrant Intrinsic Brain Activity in Patients with Autism Spectrum Disorder: Insights from EEG Microstates. Brain Topogr. 2019;32(2):295-303.

53. Malaia E, Bates E, Seitzman B, Coppess K. Altered brain network dynamics in youths with autism spectrum disorder. Exp Brain Res. 2016;234(12):3425-31.

54. Nagabhushan Kalburgi S, Whitten AP, Key AP, Bodfish JW. Children With Autism Produce a Unique Pattern of EEG Microstates During an Eyes Closed Resting-State Condition. Front Hum Neurosci. 2020;14:288.

55. Portnova GV, Ivanova O, Proskurnina EV. Effects of EEG examination and ABA-therapy on restingstate EEG in children with low-functioning autism. AIMS Neurosci. 2020;7(2):153-67.

56. Uddin LQ, Supekar K, Menon V. Reconceptualizing functional brain connectivity in autism from a developmental perspective. Front Hum Neurosci. 2013;7:458.

57. Koenig T, Prichep L, Lehmann D, Sosa PV, Braeker E, Kleinlogel H, et al. Millisecond by Millisecond, Year by Year: Normative EEG Microstates and Developmental Stages. Neurolmage. 2002 May 1;16(1):41-8.

58. Zanesco AP, King BG, Skwara AC, Saron CD. Within and between-person correlates of the temporal dynamics of resting EEG microstates. Neurolmage. 2020 May 1;211:116631.

\section{Figures}


TD (N = 15)

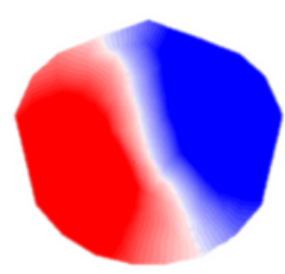

Map A

ASD-only ( $N=19$ )

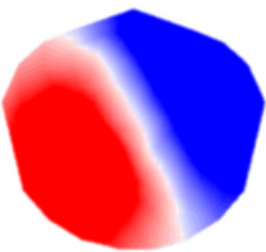

Map A

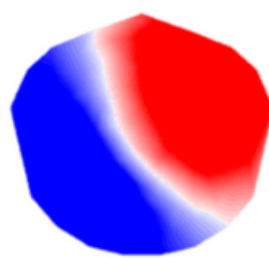

Map A

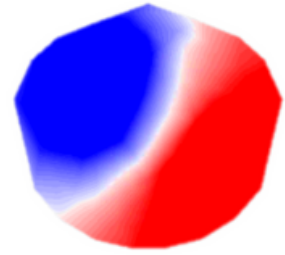

Map B

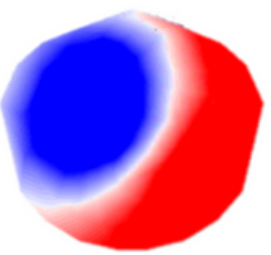

Map B

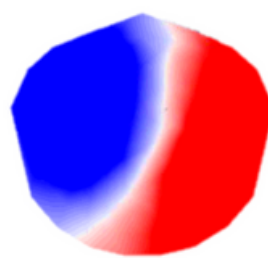

Map B

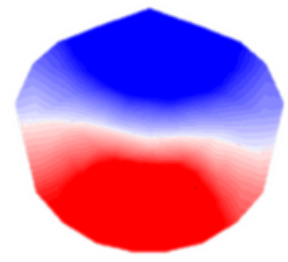

Map C

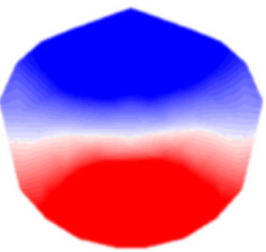

Map C

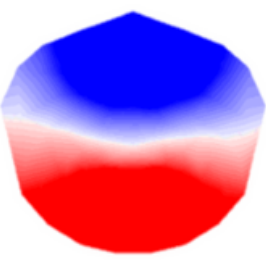

Map C

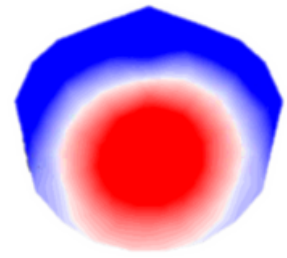

Map D

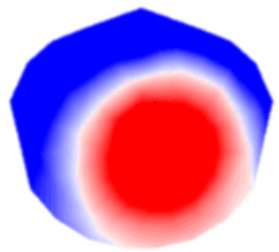

Map D

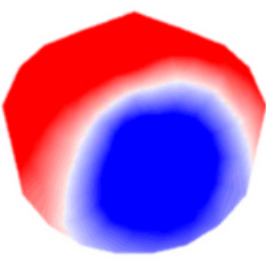

Map D

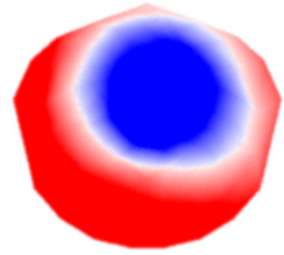

Map E

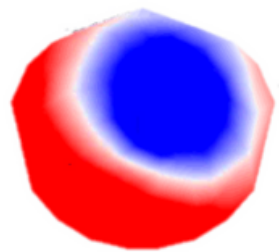

Map E

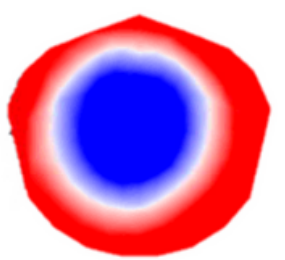

Map E

\section{Figure 1}

Template of 5 dominant microstate maps, labeled maps A, B, C, D, and E, extracted from school-aged children with ASD-only, children with ASD+ADHD and TD children separately. Polarity of the maps must be ignored. $A S D=$ Autism Spectrum Disorders; $A D H D=$ Attention Deficit/Hyperactivity Disorder; $T D=$ Typically Developing.
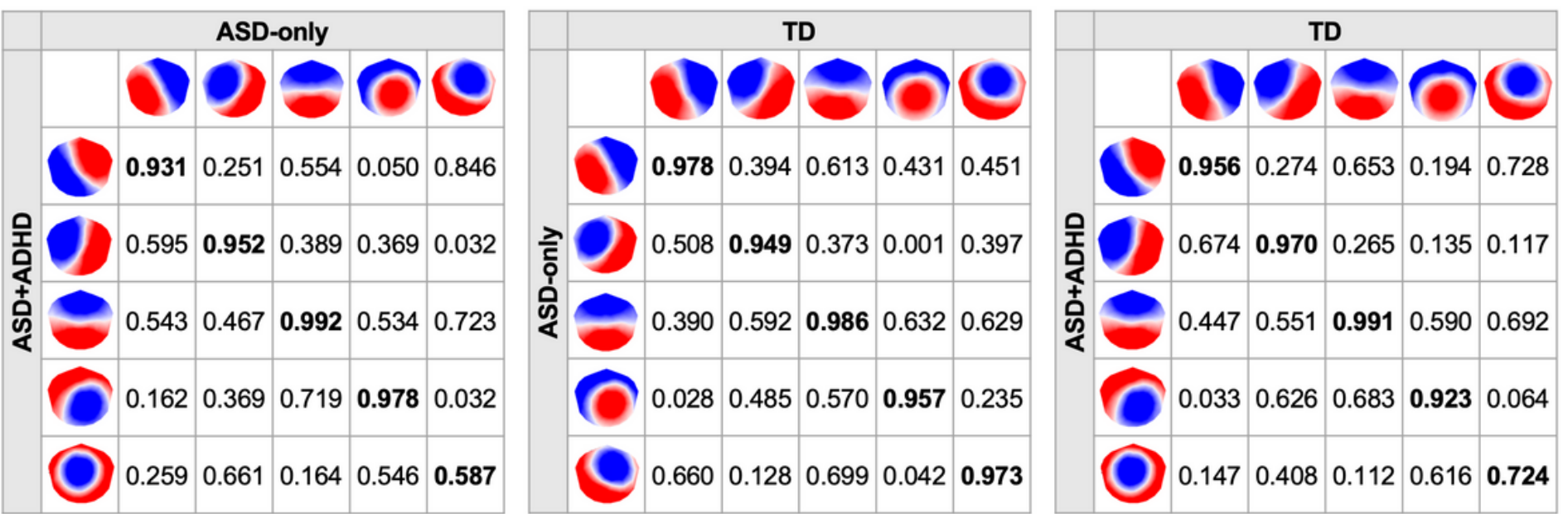
Figure 2

Pearson's spatial correlation coefficients between ASD-only, ASD+ADHD and TD groups. Polarity of the maps must be ignored. $A S D=$ Autism Spectrum Disorders; $A D H D=$ Attention Deficit/Hyperactivity Disorder; $T D=$ Typically Developing .

a)

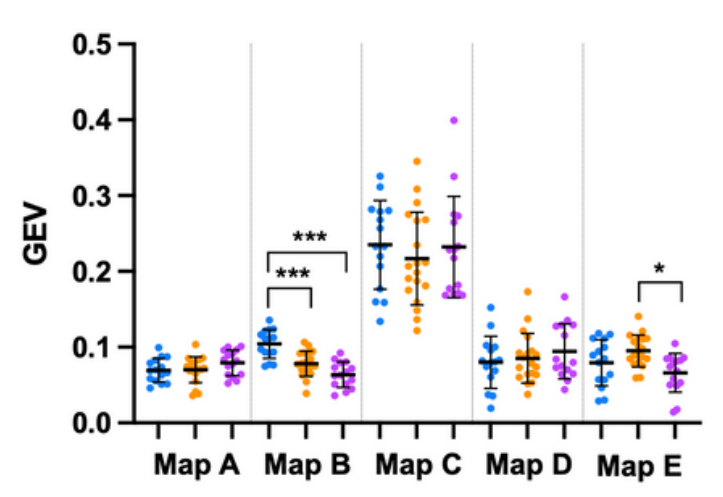

c)

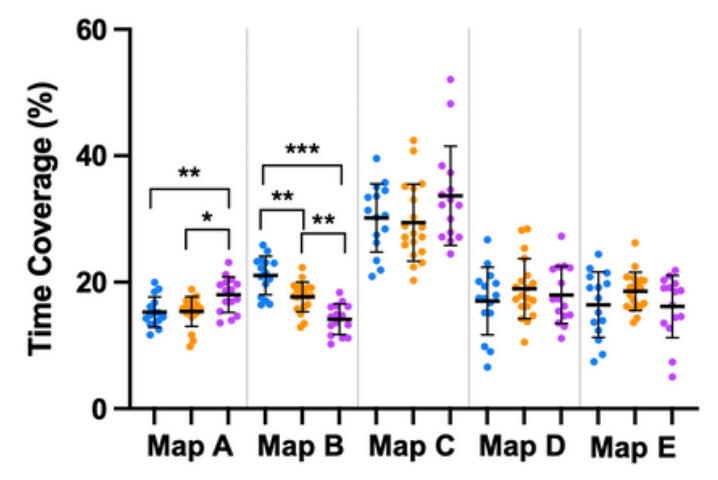

b)

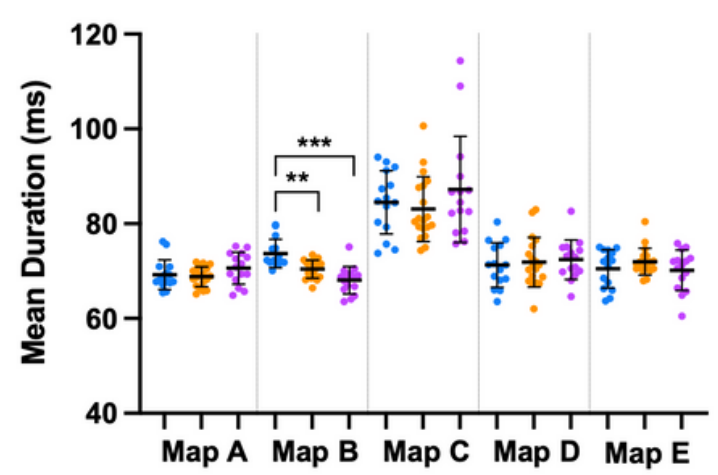

d)

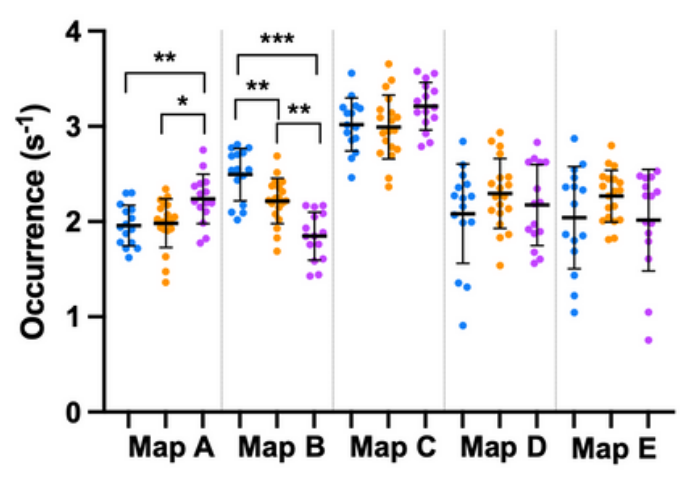

- TD ASD-only - $A S D+A D H D$

Figure 3

Temporal parameters of the microstates. Pairwise comparisons between ASD-only, ASD+ADHD, and TD groups for the temporal parameters a) Global explained variance (GEV), b) Mean duration, c) Time coverage, d) Occurrence. $P$-values are adjusted with Bonferroni correction for multiple pairwise comparisons; ${ }^{*}$-values $<0.05 ; * * p$-values $<0.01 ; * * * p$-values $<0.001$. Results are expressed as mean \pm standard deviation. $A S D=$ Autism Spectrum Disorders; $A D H D=$ Attention Deficit/Hyperactivity Disorder; $T D$ = Typically Developing. 


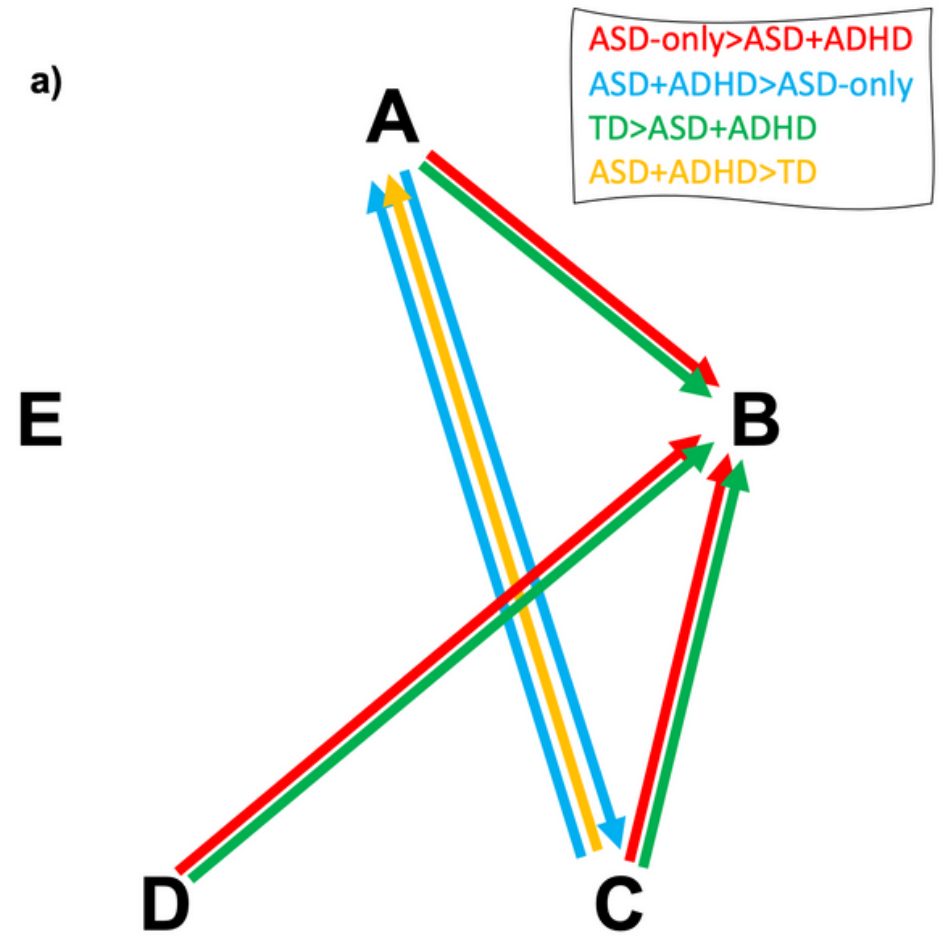

\begin{tabular}{|c|c|c|c|}
\hline & \multicolumn{3}{|c|}{ p-values for post hoc pairwise comparisons } \\
\hline & ASD-only vs ASD+ADHD & ASD+ADHD vs TD & ASD-only vs TD \\
\hline Map A $\rightarrow$ B & $0.007^{* *}$ & $0.004 * *$ & $>0.999$ \\
\hline Map A $\rightarrow$ C & $0.001 * *$ & 0.083 & 0.515 \\
\hline Map A $\rightarrow$ D & 0.080 & $>0.999$ & 0.124 \\
\hline Map A $\rightarrow$ E & 0.842 & $>0.999$ & $>0.999$ \\
\hline Map B $\rightarrow$ A & $>0.999$ & $>0.999$ & $>0.999$ \\
\hline Map B $\rightarrow$ C & $>0.999$ & $>0.999$ & $>0.999$ \\
\hline Map B $\rightarrow$ D & $>0.999$ & $>0.999$ & $>0.999$ \\
\hline Map B $\rightarrow$ E & $>0.999$ & $>0.999$ & $>0.999$ \\
\hline Map C $\rightarrow$ A & $0.001^{* *}$ & $<0.001^{* * *}$ & $>0.999$ \\
\hline Map C $\rightarrow$ B & $0.011^{*}$ & $0.003^{* *}$ & $>0.999$ \\
\hline Map C $\rightarrow$ D & $>0.999$ & $>0.999$ & $>0.999$ \\
\hline Map C $\rightarrow E$ & 0.590 & $>0.999$ & 0.134 \\
\hline Map D $\rightarrow$ A & $>0.999$ & $>0.999$ & 0.542 \\
\hline Map D $\rightarrow$ B & $0.035^{*}$ & $0.003^{* *}$ & 0.763 \\
\hline Map D $\rightarrow C$ & 0.492 & 0.204 & $>0.999$ \\
\hline Map D $\rightarrow E$ & 0.659 & $>0.999$ & $>0.999$ \\
\hline Map E $\rightarrow$ A & $>0.999$ & $>0.999$ & $>0.999$ \\
\hline Map E $\rightarrow$ B & 0.606 & 0.199 & $>0.999$ \\
\hline Map E $\rightarrow C$ & $>0.999$ & 0.110 & 0.583 \\
\hline Map E $\rightarrow$ D & $>0.999$ & $>0.999$ & $>0.999$ \\
\hline
\end{tabular}

Figure 4

Comparisons of transitions probabilities of each map between ASD-only, ASD+ADHD, and TD groups using Markov chains with a) only significant difference and b) all p-values for post hoc pairwise comparisons. $P$-values are adjusted with Bonferroni correction for multiple pairwise comparisons; ${ }^{*} p$ values $<0.05 ; * *$-values $<0.01 ; * * *$-values $<0.001$. ASD $=$ Autism Spectrum Disorders; $A D H D=$ Attention Deficit/Hyperactivity Disorder; TD = Typically Developing. 


\section{Preschoolers}

\section{TD ( $\mathbf{N}=14)$}

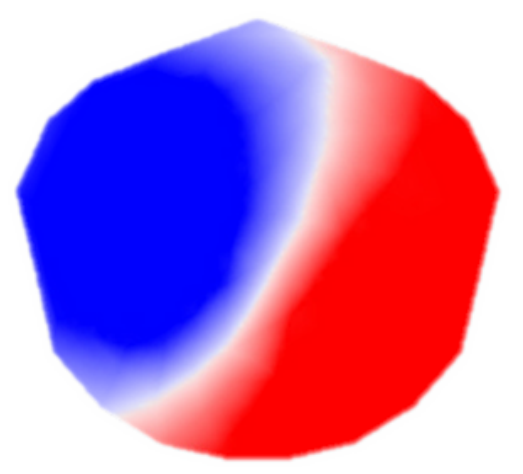

\section{ASD ( $\mathbf{N = 2 1 )}$}

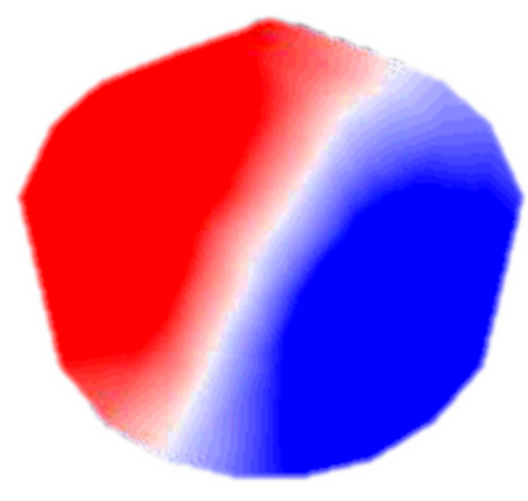

\section{School-aged}

\section{TD (N=15)}

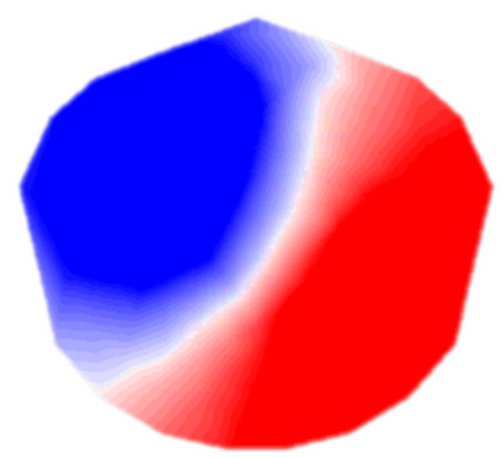

\section{ASD-only ( $\mathbf{N}=19)$}

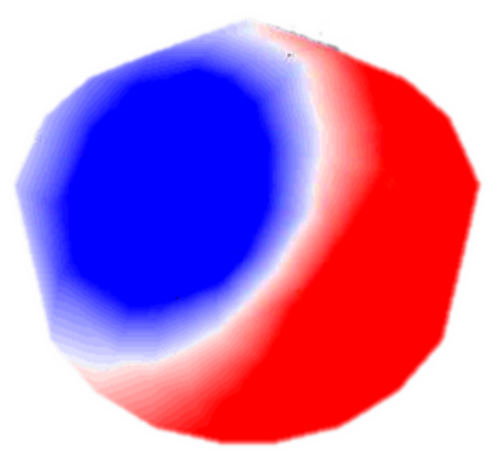

\section{ASD+ADHD ( $\mathbf{N}=15)$}

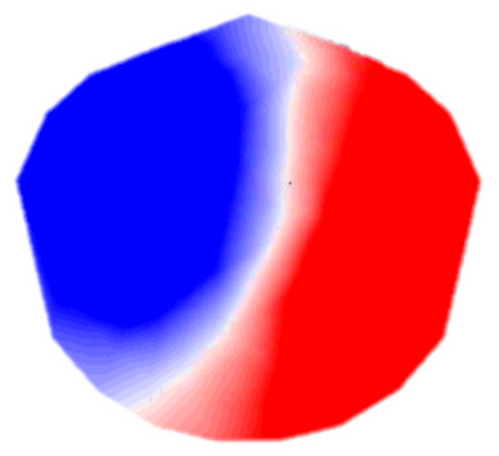

Figure 5

Template of map B extracted from preschoolers with ASD, TD preschoolers, school-aged children with ASD-only, children with ASD+ADHD and TD children separately. Polarity of maps must be ignored. $A S D=$ Autism Spectrum Disorders; $A D H D=$ Attention Deficit/Hyperactivity Disorder; TD = Typically Developing. 

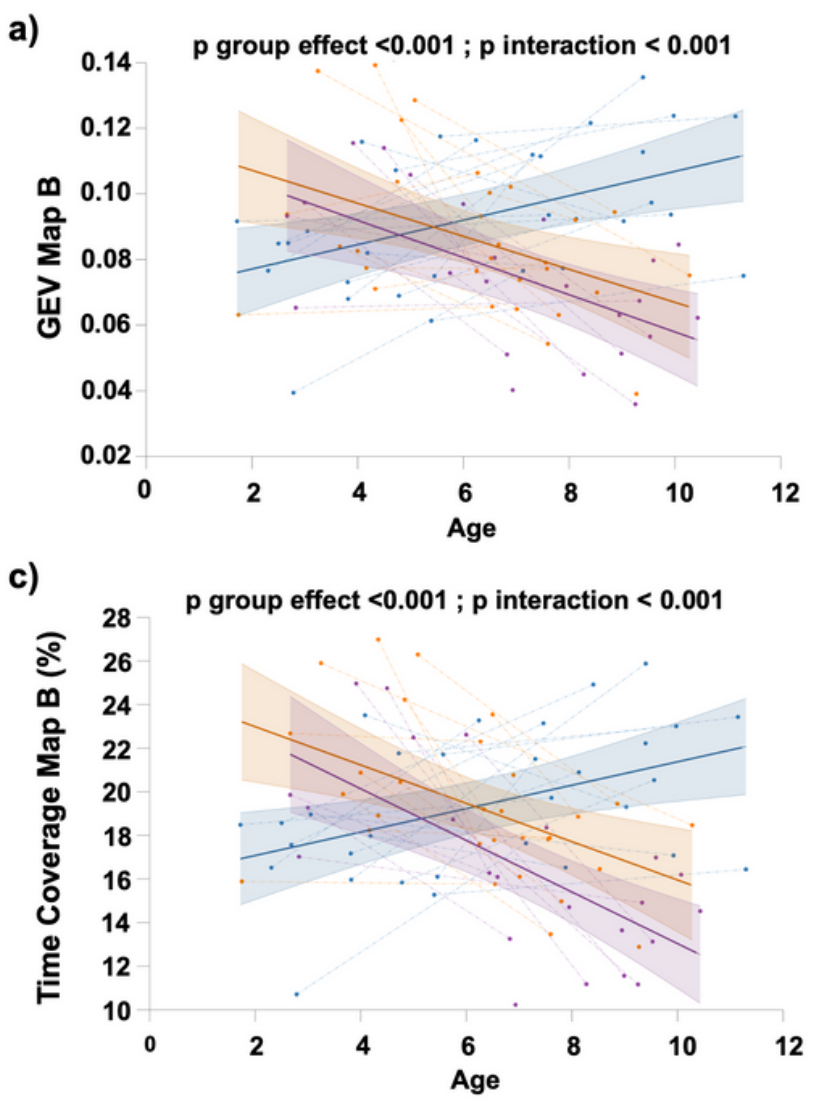

b)

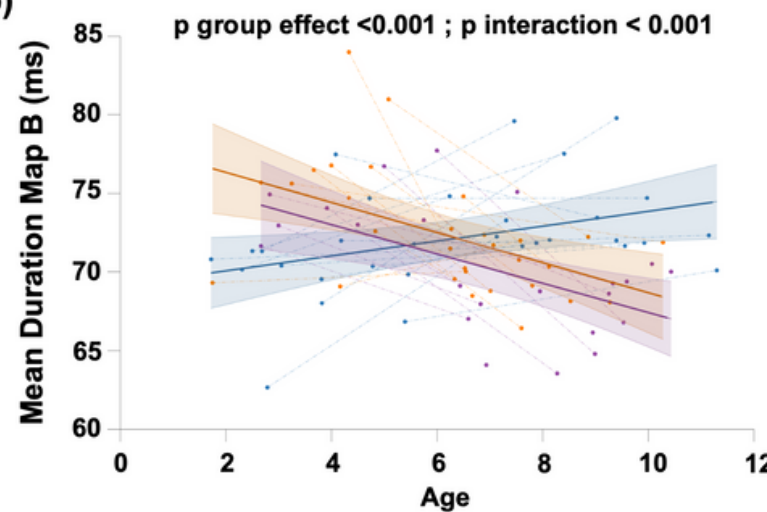

d)

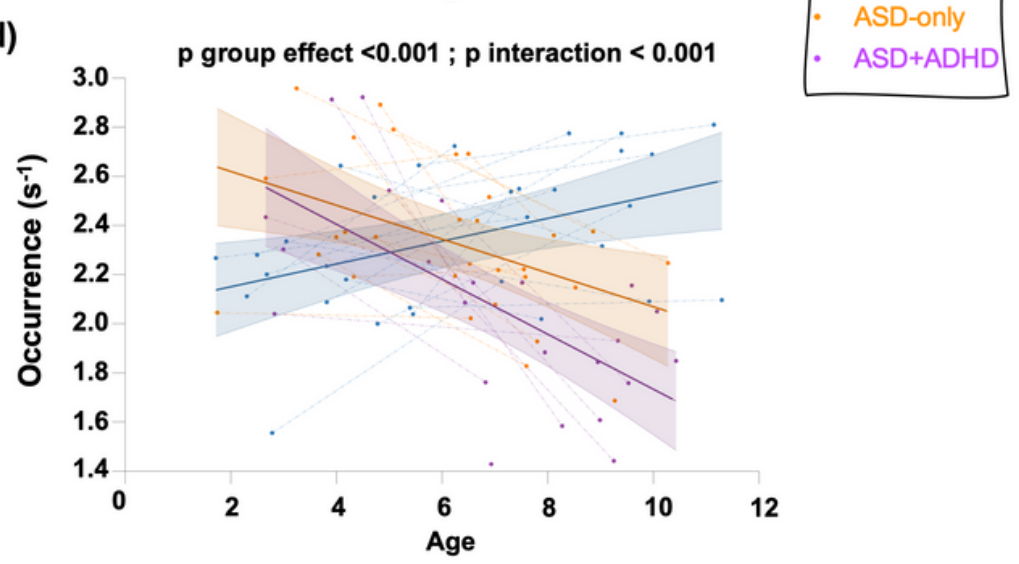

Figure 6

Exploratory longitudinal analysis for ASD-only, ASD+ADHD, and TD groups regarding map $B$ for the temporal parameters a) Global explained variance (GEV), b) Mean duration, c) Time coverage, d) Occurrence. $A S D=$ Autism Spectrum Disorders; $A D H D=$ Attention Deficit/Hyperactivity Disorder; $T D=$ Typically Developing. Results are expressed as mean \pm confidence interval. 